\title{
Gradhiva
}

Revue d'anthropologie et d'histoire des arts

\section{Des villages indigènes aux musées d'anthropologie}

De la propriété et la vente des objets rituels amazoniens

\section{Aristóteles Barcelos Neto}

Traducteur : Emmanuel de Vienne

\section{OpenEdition}

\section{Journals}

Édition électronique

URL : http://journals.openedition.org/gradhiva/602

DOI : 10.4000/gradhiva.602

ISSN : 1760-849X

\section{Éditeur}

Musée du quai Branly Jacques Chirac

\section{Édition imprimée}

Date de publication : 1 novembre 2006

Pagination : 87-95

ISBN : 2-915133-44-1

ISSN : 0764-8928

Référence électronique

Aristóteles Barcelos Neto, «Des villages indigènes aux musées d'anthropologie », Gradhiva [En ligne], 4 | 2006, mis en ligne le 03 décembre 2010, consulté le 20 avril 2019. URL : http:// journals.openedition.org/gradhiva/602; DOI : 10.4000/gradhiva.602

Ce document a été généré automatiquement le 20 avril 2019

(c) musée du quai Branly 


\section{Des villages indigènes aux musées d'anthropologie}

De la propriété et la vente des objets rituels amazoniens*

\section{Aristóteles Barcelos Neto}

Traduction : Emmanuel de Vienne

1 Le but de cet article est de contribuer à la discussion sur la division conceptuelle entre sujet et objet, dans le cas précis des transactions qui unirent les Indiens wauja ${ }^{1}$, habitant la région du Haut-Xingu en Amazonie méridionale, à différentes institutions muséologiques. La vente de rituels et/ou des objets qui sont utilisés lors de leur exécution caractérise, depuis une dizaine d'années, de nouvelles formes de relations entre Blancs et Indiens dans le parc indigène du Xingu. Partant de l'ethnographie que j'ai conduite chez les Wauja, je suggère que ces relations s'insèrent dans un contexte de capture et de domestication, de dimension socio-cosmologique, des signes extérieurs du pouvoir représenté par les Blancs.

2 La réalisation de rituels dont les Blancs sont les mécènes ou les destinataires reflète, au sein de cette même dimension, une expansion des frontières spatiotemporelles dans lesquelles les Xinguaniens peuvent manipuler des symboles de représentation politique dans le cadre de leurs relations avec les Blancs. On peut citer, à titre d'exemple, les rituels funéraires Kwarup réalisés pour les indigénistes Claudio et Orlando Villas-Boas puis pour Roberto Marinho, ancien propriétaire de la chaîne de télévision Rede Globo. En incluant cérémoniellement les Blancs, ces rituels intègrent ces relations dans un monde proprement xinguanien.

3 J'analyserai ici quelques aspects d'un cas impliquant une anthropologue américaine et le Museum of Natural History de la Smithsonian Institution, ainsi que deux projets muséologiques et ethnographiques dans lesquels j'ai joué une part active comme initiateur et maitre d'œuvre : le premier pour le Museu Nacional de Etnologia de Portugal (de septembre 1999 à décembre 2000), le second pour le musée du quai Branly à Paris (de février 2004 à juin 2005). Le projet portugais impliquait la constitution d'une collection importante et systématique et l'organisation de deux expositions de longue durée (Pais de Brito 2000 ; Barcelos Neto 2004a). Le projet français prévoyait la création d'un spectacle 
de musique et de danse des masques pour le festival de Radio France et Montpellier (Barcelos Neto 2005), qui impliquait donc l'acquisition d'une collection ethnographique de ces masques rituels. L'acquisition de ces collections dépendait, inévitablement, de la réalisation des rituels à l'occasion desquels ces objets sont produits. Mais que cela signifie-t-il de faire des rituels pour des musées?

Chez les Wauja, tout individu ou groupe, à condition qu'il dispose des ressources nécessaires, peut prendre à sa charge l'organisation d'un rituel. Les mécènes non indiens ont besoin de la médiation d'un "maitre ${ }^{2}$ " d'apapaatai. " Maître », qui se dit wekeho en wauja, est en général traduit par « celui qui prend soin d'une ressource ou de quelqu'un ${ }^{3}$ ", et apapaatai désigne les êtres pathogènes incarnés par les masques, les instruments à vent et d'autres objets rituels. Un «maître » d'apapaatai est un individu que ces êtres ont rendu malade et qui a dû satisfaire à leurs exigences alimentaires afin de stabiliser son état de santé. Ils sont représentés (au sens de représentant tel que le définit Gell [1998]) par les kawoká-mona, personnes de la communauté investies par ces mêmes apapaatai des connaissances spécifiques qui leur permettent de remplir leur rôle.

Un groupe de «maîtres » prit soin des apapaatai pour le musée du quai Branly et le Museu Nacional de Etnologia de Portugal. Il était mené par un chamane visionnaire et devin (yakapá), qui considéra que la vente des apapaatai puis leur conservation dans des musées constituait une triple opportunité : obtenir des financements pour l'achat de machines nécessaires à la vie du village, permettre aux Blancs de mieux connaître la culture wauja et offrir de la nourriture aux apapaatai via sa consommation par les kawoká-mona.

6 Le point central des transactions entre les musées et les Indiens wauja concerna la fabrication d'objets-sujets rituels (principalement des masques), qui doivent être nourris et que l'on doit faire danser et chanter avant de les vendre. Mais la vente n'efface pas complètement ni irréversiblement le fait que ces objets ont aussi un statut de personne $e^{4}$. Tant qu'ils restent intacts, le fait qu'ils sont encore quelque peu des personnes les lie à leurs « maîtres » d'apapaatai, ou mieux encore à leurs modèles spirituels pathogènes.

\section{La production de rituels d'Apapaatai}

7 À l'exception des rituels initiatiques (pohoka et Kaojatapa) et funéraires (Kwarup et Yawari), tous les rituels xinguaniens ont pour protagonistes les apapaatai - les êtres prototypiques de l'altérité.

Les apapaatai peuvent prendre sept formes rituelles de base: (1) celle de masque ${ }^{5}$ ( apapaatai onai, litt. " vêtement d'apapaatai »), (2) d'instrument à vent (flûtes, clarinettes et trompettes), (3) de chœur féminin (yamurikumã), (4) de spatule à retourner la galette de manioc et de bâton déplantoir de manioc (personnages du rituel Kukuho), (5) de Matapu et de Mapulawa (rhombe et autres personnages de la fête saisonnière du fruit Pequi), (6) de groupe de troc présidé par les hirondelles (Huluki $\left.{ }^{6}\right)$ et (7) de tronc d'arbre évidé servant d'instrument de percussion (Pulu Pulu). La forme rituelle que les apapaatai assument dépend directement et exclusivement de la forme sous laquelle ils se sont présentés aux chamanes lors des transes nécessaires à la cure d'un grand malade ${ }^{7}$.

9 Une maladie grave correspond au rapt de l'âme (principe vital, conscience) du malade par les apapaatai. En leur compagnie, elle se nourrit comme les animaux - viande crue ou pourrie, sang, herbes, feuilles, autant d'aliments qui ne font certes pas partie du régime wauja. Cette transformation alimentaire, ainsi que la compagnie ininterrompue des 
apapaatai déclenchent un processus d'animalisation du malade. Dans les rêves, c'est-à-dire dans sa condition de captive, l'âme du malade commence à acquérir la forme des apapaatai qui ont provoqué sa maladie, et en peu de temps elle sera comme un des leurs. La désanimalisation ne peut être que le fait des chamanes, qui retirent du corps du malade les substances à l'origine de sa transformation en personne-animal et révèlent quels sont les apapaatai responsables.

10 Après les révélations chamaniques, le malade (ou ex-malade si son état s'est amélioré) devient «maitre » (wekeho) des apapaatai qui le tourmentent et possède alors le droit de les fabriquer comme objets rituels, même si ce droit peut être cédé à qui montre une volonté de le faire. Le maitre devra ensuite les alimenter pendant une période allant de quelques semaines à plusieurs années, selon la nature des objets fabriqués8.

11 En ce qui concerne la mise en rituel de ses apapaatai, le malade se trouve face à quatre possibilités: ne jamais organiser de fête en leur honneur, ce qui laisse ouverte la possibilité d'une rechute provoquée par ces mêmes apapaatai; organiser pour eux de petits rituels peu après la cure chamanique; les insérer dans un rituel offert par une autre personne; conserver les apapaatai pour qu'ils soient tous fêtés en une seule fois lors d'une grande fête des masques (Apapaatai Iyãu), qui peut réunir entre 25 et 50 personnages rituels?.

12 Je me pencherai ici sur le cas d'un Apapaatai Iyãu, le seul rituel susceptible de donner lieu à la fabrication d'un grand nombre de masques, condition nécessaire à la constitution d'une collection ethnographique systématique.

13 Au début des années 1990, l'actuel chef wauja, Atamai, a souffert d'une très sérieuse infection oculaire. Les deux yakapá les plus réputés de Piyulaga, le village wauja, furent chargés d'identifier les agents pathogènes en cause. Ils découvrirent et nommèrent Kawoká (flûte de bois), Kawoká Otãi (petit de Kawoká), Yamorikumã et Makaojeneju (deux types de femmes monstrueuses), Tankwara Yanumaka (Jaguar clarinettiste), Atujuwá Yanumaka (Jaguar "vêtu » d'un tourbillon), Ewejo (Loutre), Yuma (Poisson-chat à queue rouge ${ }^{10}$ ), Tukujen (une espèce de Pigeon), Kukuho (une espèce de Larve), Kagaapa (une espèce de Poisson), Yukuku (une espèce d'Arbre), Nukuta Pitsu Run Run Run (Archer), Kapulu (Saki noir), une espèce de poisson vêtu de Kuwahãhalu, et sept espèces d'apapaatai revêtus de Sapukuyawã (un type de masque générique).

14 Après les révélations des chamanes, Atamai est donc devenu le «maître » des apapaatai cités. Une fête fut organisée en l'honneur de Kukuho pendant un séjour d'Atamai dans un hôpital de Brasília, puis une autre pour Ewejo dès son retour à Piyulaga. Deux ans plus tard ce fut le tour de Tankwara et de Kagaapa. Les dix-neuf apapaatai restants furent « rangés » dans l'attente d'une occasion appropriée d'organiser pour eux une cérémonie. Cette occasion apparut de façon inespérée - et plutôt originale - pour Atamai sous la forme d'une demande de la boutique Artíndia ${ }^{11}$ de Brasília. Elle souhaitait que les Wauja effectuent une "démonstration" de leur grand rituel des masques, alors presque inconnu. Un récit d'Atamai daté de 2000 , dont je reproduis ici un extrait, situe cet événement dans le contexte de sa longue maladie et de la production rituelle des masques qui lui est associée.

«Là-bas, à Brasília, je me suis senti encore plus mal. J'ai cru que j'allais mourir. Je n'avais plus d'appétit, je ne mangeais plus rien du tout, et du coup je devenais très faible. Je croyais que les apapaatai étaient avec moi. Après j'ai découvert que Tankwara et Atujuwá qui étaient avec moi étaient [des apapaatai] de Yanumaka [Jaguars]. Ils étaient tous les deux avec moi. C'est pour ça que dans mes rêves je 
mangeais la nourriture des jaguars : des pécaris, des cerfs, des tapirs. C'étaient ces jaguars qui me donnaient ces animaux pour que je les mange. Alors à cause de ça je n'avais pas faim.

Les gens au village ont appris que j'allais de plus en plus mal. Alors mes Kawokámona ont demandé à ma fille s'ils pouvaient faire pour moi la fête de Kukuho, même si j'étais loin. Ma fille a dit que c'était d'accord, et elle a accepté de fournir toute la nourriture nécessaire pendant que j'étais à Brasília. C'est comme ça qu'ils ont fait pour moi la fête de Kukuho.

Quand la fête s'est terminée ici, à Piyulaga, la même nuit j'ai rêvé que certaines "personnes" [apapaatai] me parlaient:

“- On s'en va, maintenant que se termine ce qu'on voulait."

Et quand je me suis réveillé je me sentais mieux. Je n'avais déjà plus ces douleurs très fortes que j'avais avant. J'avais juste quelques migraines.

Quand je suis rentré au village, on m'a dit : - Oncle, on a fait apapaatai [Kukuho] pour toi."

Je me suis alors dit: "C'est donc pour ça que je vais mieux." J'ai été très touché par ce qu'ils avaient fait, par le souci qu'ils avaient de mon état. Ils ont fait ce qu'il fallait pour que j'aille mieux. Alors je suis resté ici au village. J'allais déjà un peu mieux, mais je ne pouvais pas travailler comme avant, ni rester longtemps au soleil. Peu après mon retour, ils ont fait pour moi Ewejo [Loutre]. Ils m'ont appelé et m'ont dit qu'ils allaient faire apapaatai pour que j'aille mieux. Puis ils l'ont fait. Après la fête d'Ewejo je n'ai plus jamais pêché dans mes rêves, ni mangé du poisson cru.

Après quelque temps ils ont fait Tankwara. Ce sont eux qui m'aident maintenant. Ils ne laissent pas les autres apapaatai me faire du mal. Maintenant je vais bien. Il n'y a que mon œil qui de temps en temps me fait un peu souffrir.

Mais mes rêves avec les "personnes" [apapaatai] n'avaient pas cessé complètement. Je rêvais encore beaucoup de ceux qui m'avaient rendu malade. Jusqu'à ce que les gens d'Artíndia à Brasília nous demandent de leur montrer la fête des apapaatai.

J'en ai alors parlé à mes frères et mes neveux [qui dans ce cas sont ses kawoká-mona ], qui ont accepté de faire la fête. Les gens d'Artíndia sont venus, ont regardé, pris des photos des danses, pris les masques pour la boutique et ne sont plus jamais revenus.

C'est moi qui ai pris à ma charge cette fête des masques pour Artíndia. J'ai demandé à tout le monde dans le village s'ils avaient des apapaatai et s'ils avaient envie de faire leur fête. Ceux qui avaient déjà des apapaatai ont décidé de leur faire des fêtes plus tard. Il n'y a donc que moi qui ai offert mes apapaatai pour que soit faite la grande fête. »

Les événements évoqués dans ce récit couvrent une période d'environ sept ans, du départ d'Atamai pour Brasília à la réalisation du grand rituel des masques (Apapaatai Iyãu) pour Artíndia en août 1997. En contrepartie les Wauja ont demandé de l'aide pour ouvrir une piste d'atterrissage pour petits avions, qui a consisté en l'envoi des machines nécessaires aux travaux. Il est important de remarquer qu'Atamai associe de façon directe et sans équivoque sa guérison à la réalisation du rituel, et qu'il insiste sur le fait que les étrangers peuvent commander ou sponsoriser une fête " authentique » d'apapaatai ${ }^{12}$.

L'origine d'une telle organisation du rituel, qui lie ce dernier à des institutions comme des musées ou des boutiques de renom, n'est pas si récente. Elle remonte au moins à une vingtaine d'années. Et si nous associons à ce phénomène l'existence d'un commerce important de la culture matérielle wauja, il nous faut prendre en compte la grande collection d'Harald Schultz qui, en 1963, réunit 664 pièces pour le musée Paulista de l'université de São Paulo.

L'anthropologue américaine Emilienne Ireland voulut réunir une collection de masques alors qu'elle menait en 1983 une enquête de terrain financée par le National Museum of 
Natural History de la Smithsonian Institution. Mais l'acquisition des pièces se révéla plus difficile qu'il n'y semblait à première vue. Il s'avéra impossible pour Ireland d'acheter les masques que les Wauja gardaient pour les brûler ensuite au cours du rituel. Elle ne put pas davantage commander les masques à un artisan, puisque les Wauja considéraient leur fabrication «simplement pour les vendre» comme un manque de respect envers les apapaatai.

«Because the sponsor of a given ceremony is supposed to display in his house paraphernalia associated with the ceremony he has sponsored, it would not have been possible for me to collect a mask that had been used on a ceremony commissioned by a Waura without going against local custom and public opinion. of course, it is always possible to find individual Waura who are willing to sell to outsiders items that the Waura say it is wrong to sell, but this would have create bad feelings. The only reasonable solution was to sponsor a ceremony myself, which would automatically make me the rightful owner of the ceremonial paraphernalia. I had planned to do this as a purely economic transaction, and applied to the Smithsonian for a grant to sponsor the atujuá ceremony in this fashion. » (Ireland $1985: 16-17)$

18 La seule possibilité encore ouverte à Ireland pour posséder ces objets était donc de commander elle-même un rituel de masques. Mais ce projet connut une péripétie inattendue.

Pendant que les Wauja attendaient la venue de São Paulo d'une partie des matières premières nécessaires à la fabrication des masques pour le National Museum of Natural History de la Smithsonian Institution, Ireland tomba gravement malade et fit appel aux soins d'un chamane du village (Ireland 1985 : 18). D'après le diagnostic chamanique, sa maladie était provoquée par les apapaatai Sapukuyawã et Kuwahãhalu, dont elle devint logiquement «maitre ». En tant que tel, elle put sans problème organiser le rituel propre à ces masques et donc les acquérir au profit du musée. Du fait de sa maladie elle acquit directement un «droit rituel » sur les apapaatai. Mais comme celle-ci n'avait pas été provoquée par les grands masques circulaires Atujuwá, elle n'eut pas le droit de les faire fabriquer pour son rituel de guérison ${ }^{13}$.

20 J'ai eu connaissance de la tenue du rituel Apapaatai Iyãu pour la boutique Artíndia avant même mon premier séjour à Piyulaga, en mars 1998. Mais je n'appris les détails de la négociation qu'en mars 2000, lorsque j'abordai la question avec Atamai. Je voulais assister à ce rituel depuis 1998, mais souhaitais qu'il ait lieu spontanément, hors du cadre d'une transaction purement commerciale. Ceci s'avéra impossible.

La conversion des apapaatai de biens métaphysiques en artefacts rituels est avant tout une décision personnelle, ou plutôt une décision de l'individu qui détient des apapaatai qui n'ont pas encore reçu de fête. S'il se décide à en organiser une, il peut alors solliciter, grâce à la médiation du chef, dans ce cas le "maître du village » (putakanaku wekeho), l'aide de la communauté. Ces négociations internes sont délicates et leur succès dépend du soutien appuyé des amunaw (" nobles »/ chefs). La possession d'un grand nombre d' apapaatai n'est donc pas une condition suffisante à la réalisation du rituel Apapaatai Iyãu.

En même temps que je m'employais à acquérir des masques pour le Museu Nacional de Etnologia de Portugal, je m'intéressais à l'exécution authentique du rituel des masques. Je supposai d'abord que le processus « authentique » qui guide son exécution serait altéré par des motivations commerciales et qu'en commandant les masques je me trouverais face à une simple démonstration de danse, et non face à un rituel. Il ne me fallut que quelques semaines pour m'apercevoir que je me trompais largement. 
pendant la dernière semaine de juin 2000 que j'ai évoqué avec Atamai la possibilité de réaliser un Apapaatai Iyãu. "C'est fini depuis cette fête » (pour Artíndia) fut la réponse que j'entendis à ma demande d'acheter des masques pour la collection. Il voulait dire en fait qu'il ne lui restait plus de masques. "Je vais voir si quelqu'un en a encore", dit Atamai, en cherchant à ne pas décevoir mes attentes. Atamai porta l'affaire devant le « maître du village » et les autres amunaw, qui en discutèrent une semaine entière. On put alors vérifier que seul l'amunaw Itsautaku disposait d'assez d'apapaatai pour réaliser un Apapaatai Iyãu. Il accepta immédiatement d'organiser un rituel pour ses apapaatai, à la condition que ses kawoká-mona acceptent de prendre en charge leur fabrication. Face à l'accord unanime des kawoká-mona, la question fut discutée une fois de plus au «conseil des amunaw ». Après plusieurs semaines, ma commande devint ainsi un rituel.

Bien que le rituel ait été initialement motivé par une commande, cette circonstance s'avéra rapidement accessoire. Peu après les premières discussions sur le paiement des masques et l'organisation de l'Apapaatai Iyãu, les Wauja cessèrent d'évoquer le destin muséologique des masques, comme s'ils y étaient indifférents. Gagnés par la joie de la fête, les Wauja dotèrent l'Apapaatai Iyãu d'une signification propre. La réalisation du rituel ne dépendait déjà plus d'une cause extérieure aux Wauja. À cette occasion, je pus collecter un certain nombre d'informations sur la fête donnée « pour » Artíndia.

La raison invoquée pour la réalisation de l'Apapaatai Iyãu d'août 1997 a été élaborée a posteriori par les Wauja. Ils affirment qu'ils firent la fête " pour Atamai, c'était sa fête, pour qu'il aille mieux, pour qu'il ne souffre plus, pour qu'il rêve bien ». Atamai lui-même affirme dans son récit : «Après cette grande fête mon état s'est vraiment amélioré. Je me suis senti si fort. Je me suis vraiment senti bien. » C'est comme s'il avait attendu depuis longtemps cette fête, dont le moment opportun se révéla être précisément celui où Artíndia manifesta son intérêt pour les masques. Pour cette raison, la fête n'eut pas pour objectif d'obtenir une piste d'atterrissage - qui fut d'ailleurs finalement dégagée sans l'aide d'Artíndia. Le point de vue qui prédomine est en dernier ressort celui de la pathologie et de la guérison ou, pour le dire autrement, en l'absence de visée thérapeutique il n'y a pas lieu de faire les rituels de masques et d'instruments à vent.

\section{La vie occulte des objets et la rétention du statut de personne}

En octobre 2004, je me rendis à nouveau à Piyulaga pour mener un travail de terrain et pour proposer aux Wauja d'organiser une autre fête Apapaatai Iyãu. Mon objectif cette fois était triple: réaliser un film ethnographique, réunir une collection ethnographique de masques pour le musée du quai Branly et adapter le rituel pour en faire un spectacle que dix-sept Wauja représenteraient au festival de Radio France et Montpellier en juin 2005. Quelques Wauja mirent à disposition leurs apapaatai qui n'avaient pas encore fait l'objet d'une fête pour pouvoir accomplir un Apapaatai Iyãu, qui eut lieu en mars 2005. De même qu'en 1997 avec Artíndia-FUNAI et qu'en 2000 pour le Museu Nacional de Etnologia, la décision de faire un Apapaatai Iyãu pour le musée du quai Branly puis pour le festival de Radio France déclencha immédiatement la constitution d'un réseau de services rituels. Ceux-ci consistèrent en dons obligatoires de nourriture équilibrés par des cadeaux: marmites de céramique, flèches, longs colliers de perles et autres objets d'apparat ${ }^{14}$. 
elle procure aux apapaatai une satisfaction esthétique qui supprime leur agressivité pathogène et oriente l'action rituelle vers la guérison. Il revient à la beauté de contribuer au succès de la thérapie. continue, ce qui obligea à mettre en service une deuxième. Les trois autres restèrent rangées $^{15}$. Interrogé sur la possibilité de vendre ses marmites, Atamai opposa un refus
catégorique : «C'est le paiement de Tankwara, je ne peux pas manquer de respect à celui rangées ${ }^{15}$. Interrogé sur la possibilité de vendre ses marmites, Atamai opposa un refus
catégorique : «C'est le paiement de Tankwara, je ne peux pas manquer de respect à celui qui m'aide. Si je fais ça, je meurs.»

Les apapaatai et tout ce qui s'y rapporte sont entourés d'un soin minutieux. Quand on décide de leur donner corps sous la forme d'objets rituels, la première disposition morale symétrique qui émerge entre le « maître » du rituel et ses kawoká-mona est celle qui réunit sous une même appellation la honte et le respect. Le rituel génère de plus en plus d'obligations entre les participants, caractéristique reconnue comme sa principale vertu morale. Il ne s'agit pas simplement de produire pour échanger ou vendre (que ce soit entre Indiens ou entre Indiens et Blancs), mais de réveiller la vie occulte des objets ou, plus précisément, de réveiller les apapaatai affamés à la vie d'objets-sujets. Toute la nourriture dont la production est exigée par le rituel est destinée à satisfaire les apapaatai . Nous aborderons quelques détails de ce processus de réveil et comprendrons alors comment l'offrande d'aliments se situe derrière la rétention par les objets d'origine rituelle de leur statut de personne.

trouvai par hasard face à trois marmites kamalupo très impressionnantes, protégées par de vieux tissus poussiéreux. Elles faisaient partie d'un ensemble de cinq marmites offertes au chef Atamai par ses Kawoká-mona Tankwara. Chacune d'elles correspondait à l'un des cinq tubes qui constituent invariablement la formation des clarinettes Tankwara, chaque tube correspondant à son tour à un Kawoká-mona Tankwara.

aux plus durables, qui se superpose à deux autres échelles: celle de la dureté des matériaux utilisés et celle de la difficulté et du temps de fabrication. Au pôle inférieur de la dureté se trouvent les masques de paille (apapaatai onai) et au pôle supérieur les flûtes en bois (Kawoká). Ces dernières sont au centre du système rituel et à l'origine de la fabrication d'autres objets moins solides et moins durables. Les Kawoká, par leur grande permanence dans le temps et le fait qu'elles conservent longtemps leur statut de personne, peuvent mettre en jeu une chaîne d'activités productives allant de la plantation d'un jardin à la construction d'une maison et des silos attenants destinés à recevoir la farine de manioc issue du jardin.

En 1999, après que fut terminée la grande amunaw opona (maison du chef), eut lieu à Piyulaga un Iyeju Tankwara, grand rituel dé-dié aux cinq "maitres » des Tankwara en échange de la nourriture qu'ils ont offerte à leurs kawoká-mona pendant des mois ou des années. Ces derniers offrirent en cette occasion vingt-cinq marmites de céramiques aux cinq «maîtres » des Tankwara. Celles d'Atamai, peintes de motifs extrêmement raffinés, comptaient parmi les mieux faites.

Des cinq marmites d'Atamai, l'une se fendit en avril 2000 après des mois d'utilisation Dans cette déclaration il place implicitement ses kawoká-mona (ceux qui ont fabriqué les marmites) dans une situation d'intermédiaires et indique que l'aide reçue le fut de Tankwara lui-même, plus précisément des Jaguars à l'origine de sa maladie. Il invoque 
également un principe élémentaire de la relation aux apapaatai - la peur-respect (soit le risque de tomber gravement malade) - pour renforcer le point de vue de l'interdiction de vente. La désapprobation ne serait donc pas tant manifestée par les Kawoká-mona que par les Jaguars. De ce fait, les marmites n'appartiennent pas à Atamai, mais bien aux Tankwara , puisque ce sont elles qui permettent aux Jaguars de consommer des aliments cuits. En juillet 2000, un mois après l'épisode des marmites, nous nous trouvions à discuter, Atamai et moi, de sa maladie et des travaux en cours de ses Kawoká-mona Tankwara. Il me dit alors de but en blanc: "J'ai aussi eu Ewejo [Loutre]. Il y a longtemps, elles m'ont beaucoup aidé. Tu veux les voir?» De l'air de quelqu'un se rappelant subitement une chose oubliée depuis longtemps, il se dirigea vers un sombre recoin de la maison avec une lampe de poche, le fouilla et rapporta, en le traînant au sol, un énorme sac en plastique couvert de poussière, de suie et de toiles d'araignée dont il entreprit de vider le contenu. Apparurent huit masques de Loutre (Ewejo) fabriqués à son retour de l'hôpital de Brasília. Ils constituaient le groupe de personnages du deuxième rituel d'apapaatai que firent les Wauja pour Atamai après sa maladie. Le premier, Kukuho, avait eu lieu pendant son séjour à l'hôpital. En juillet 2000, ces masques avaient donc près d'une décennie.

En regardant les huit masques disposés sur le sol, Atamai répéta : "Ces Loutres m'ont beaucoup aidé. » Je lui demandai si elles l'aidaient encore. Atamai répondit que non, qu'elles - les Loutres qui l'avaient rendu malade - s'en étaient déjà allées, mais qu'un jour elles (en tant que masques) seraient brûlées.

Quelque temps après le rituel des masques, les huit Kawoká-mona Ewejo d'Atamai lui fabriquèrent près d'une vingtaine de paniers de transport, qu'il suspendit en ligne au plafond de sa maison. Une telle disposition a certainement causé une vive impression aux étrangers qui ont visité Piyulaga à l'époque. D'après Atamai, ceux-ci lui demandaient avec insistance de leur vendre ne serait-ce qu'un panier. Il refusa de façon systématique. Les paniers furent utilisés les uns après les autres par les femmes de la maison. Le dernier fut décroché du toit près de deux ans plus tard. De la même façon que sa amunaw opona (maison du chef), les paniers sont les signes visibles de son alliance avec les apapaatai, avec les Kawoká-mona et par extension avec la communauté.

37 Après m'avoir raconté l'histoire des paniers, Atamai me demanda si je souhaitais emporter les huit masques pour les donner au musée (le Museu Nacional de Etnologia de Portugal). L'immense surprise que me causa cette proposition devint ensuite une question: pourquoi les masques, à la différence de certains paniers et des marmites de céramique, peuvent-ils être vendus à des musées? La réponse implique la prise en compte de trois aspects fondamentaux : la fonction, la fabrication et la possession des objets considérés. Les masques perdent leur fonction après avoir été utilisés lors du rituel. Mais, dans certains cas exceptionnels comme celui d'Atamai, les masques peuvent, avant d'être brûlés, connaître une brève collaboration avec des objets rituels qui leur sont hiérarchiquement supérieurs.

38 Après le rituel Ewejo, les masques ont été rangés dans la maison, sans que la relation d'Atamai avec ses Kawoká-mona ne cesse pour autant. Il continua de leur offrir de la nourriture de temps en temps. Deux ans plus tard, les Kawoká-mona Tankwara décidèrent d'exécuter le rituel de clarinettes pour Atamai, rassemblant ainsi un nouveau groupe de production rituelle auquel les huit Ewejo s'associèrent en tant que collaborateurs. Leur supériorité hiérarchique comme leur tendance à rester longtemps des personnages rituels font des clarinettes des pôles d'attraction pour les masques et les situent dans le contexte plus vaste de l'exécution d'un rituel pour un même «maître». En d'autres 
termes, si Atamai n'avait pas possédé les clarinettes, jamais les masques n'auraient travaillé dans ses jardins. Ils auraient tout au plus fabriqué quelques paniers.

Les marmites, les paniers ainsi que d'autres artefacts intègrent la chaîne de production et de paiements rituels dont j'ai fait mention au début de cette sous-partie. Ces objets sont des indices de la distribution des personnes humaines et non humaines (Gell 1998), distribution qui dans le cas présent s'organise en deux temps. En premier lieu, les apapaatai fragmentent l'âme du malade, chaque fragment correspondant à un apapaatai ravisseur que l'on familiarise par l'offre de nourriture à un kawoká-mona. La première grande distribution a donc lieu au niveau des membres de la communauté. En second lieu, les kawoká-mona fabriquent des artefacts rituels (le plus souvent des masques et des instruments à vent) qui leur permettent d'actualiser la capacité d'agir des apapaatai, que l'on oriente ensuite vers la culture du jardin, la fabrication de marmites, de paniers, de balles de pequi, de pièges de pêche, de spatules à galette et de déplantoirs de manioc. Par conséquent la deuxième grande distribution a lieu au niveau des artefacts.

Ces artefacts prennent part aux relations qui permettent la production du rituel au même titre que les membres de la communauté. Ils ne sont donc pas de simples instruments de travail, mais bien des personnes qui travaillent. Les artefacts dont Atamai interdit rigoureusement la vente sont véritablement des personnes, statut qui leur est conféré autant par les apapaatai que par les kawoká-mona qui les ont fabriqués. C'est la raison pour laquelle la vente aux musées ne concerne que les objets rituels qui sont destinés à être brûlés, oubliés ou détruits par le temps, autrement dit ceux qui connaissent, après l'exécution du rituel, un long processus de perte du statut de personne. Les masques sont par excellence de ce type d'objets : ils meurent de faim ou sont consumés par le feu. À l'opposé il est des objets rituels qui sont hyper-rétenteurs de ce statut de personne, comme les flûtes Kawoká, les clarinettes Tankwara et le tronc évidé Pulu Pulu, qui en principe reçoivent de la nourriture pendant des années ou des décennies. Ils feront très difficilement l'objet d'une transaction monétaire sans que soit effacée une part de ce qui en fait des personnes.

41 Si l'on considère le régime de distribution et de rétention des propriétés de la personne pour les objets rituels, la décision des Wauja d'organiser un Apapaatai Iyãu, de fabriquer les objets nécessaires à son exécution pour les abandonner ensuite en France est loin de constituer une position hétérodoxe. Il s'agit pour eux d'une transaction avec des institutions où leurs déjà quasi-objets seront presque seulement des objets.

\section{NOTES}

* Ce texte est fondé sur le cinquième chapitre de ma thèse de doctorat (Barcelos Neto sous presse.a) ainsi que sur des travaux de terrain conduits dans le village wauja de Piyulaga en octobre 2004 et mars 2005 et à Montpellier en juillet 2005. Je tiens à manifester ma reconnaissance aux Wauja, et en particulier à Atamai, Itsautaku, Aulahu, Kamo, Yanahin, Kuratu et Hukai. Les recherches de terrain ont été financées par le gouvernement de l'État de Bahia et par le Funpesquisa/UFSC (année 1998), par le Museu Nacional de Etnologia de Portugal (année 
2000), par la FAPESP (années 2001, 2002, 2004 et 2005) et par le musée du quai Branly (année 2005). La CAPES et la FAPESP m'ont accordé des bourses d'étude à différentes étapes de ma recherche. Je remercie Lux Vidal, Maria Rosário Carvalho, Michael Heckenberger, Rafael Bastos, Carlos Fausto et Bruna Franchetto, qui ont encouragé mes recherches dans le Haut-Xingu.

1. Le lecteur rencontrera dans la littérature ethnologique le terme "Waurá», ethnonyme consacré depuis la première publication sur le Haut-Xingu (Steinen 1886). J'ai choisi la graphie « Wauja », qui correspond à l'auto-ethnonyme. Les Wauja parlent une langue arawak et habitent depuis plus d'un siècle sur la rive droite du fleuve Batovi, dans la région occidentale des formateurs du fleuve Xingu, dans l'État du Mato Grosso, au Brésil central. Leur présence dans le Haut-Xingu est cependant bien plus ancienne. Des recherches archéologiques récentes datent l'arrivée dans la région des ancêtres des Wauja autour $\mathrm{du}_{\mathrm{Ix}} \mathrm{e}^{\mathrm{e}}$ siècle (Heckenberger 2001). Dès le début $\mathrm{du} \mathrm{XVIII}^{\mathrm{e}}$ siècle se mit en place dans cette zone un système social multiethnique qui intègre, outre les Wauja, neuf autres groupes aux affiliations linguistiques variées - Mehinaku et Yawalapiti (Arawak) ; Kuikuru, Kalapalo, Matipu et Nahukwá (Caribe) ; Kamayurá (Tupi-Guarani), Aweti (Tupi) et Trumai (langue isolée). La population wauja compte approximativement 360 individus, parmi lesquels environ 300 vivent dans un village circulaire organisé autour d'une place centrale et d'une " maison des flûtes ». Les 60 autres vivent dispersés dans d'autres villages du Haut-Xingu, au poste indigène de Batovi, dans une exploitation agricole de la rivière Von den Steinen et dans des villes voisines du parc du Xingu.

2. Traduction du portugais dono. $\mathrm{NdT}$.

3. Pour une discussion approfondie de la notion xinguanienne de «maître ", voir Viveiros de Castro (2002).

4. «Statut de personne » est une traduction du terme anglais personhood.

5. Pour une étude détaillée de la typologie et de l'iconographie des masques wauja, voir Barcelos Neto (2004b).

6. Le choix des minuscules ou des majuscules pour la graphie des non-humains renvoie à la distinction entre les personnes-animaux (dorénavant Animaux) et les animaux-animaux (dorénavant animaux). Ainsi, «jaguar » yanumaka renvoie à l'animal de l'espèce Panthera onca, alors que «Jaguar» (Yanumaka) fait référence à une personne-jaguar. Les objets les plus prototypiques sont aussi écrits avec une majuscule (par exemple Tankwara, la clarinette ; Kawoká, la flûte de bois; Sapukuyawá, un masque que peuvent revêtir tous les animaux, certains phénomènes naturels et certaines plantes).

7. Sur les processus visionnaires et divinatoires chez les Wauja, voir Barcelos Neto (2001a et b, 2002 et 2006).

8. Les offres d'aliments et la durée de vie des objets rituels ont une incidence directe sur la façon dont les Wauja pensent la permanence des apapaatai. Dans un travail récent (Barcelos Neto sous presse.b), j'analyse les stratégies et les conflits auxquels donne lieu l'entretien des objets, ainsi que la place qu'il occupe dans les cycles rituels biographiques.

9. La performance d'un Apapaatai Iyãu se déroule sur une «scène » bien définie : l'enekato (la grande place centrale du village), dont la surface à Piyulaga s'élève à $15540 \mathrm{~m} 2$. Une petite partie de cette surface a pour nom enekutaku et se situe juste devant l'entrée du kuwakuho (maison des flûtes). Les Wauja traduisent correctement enekutaku par «le centre ». C'est là que se déroulent d'ailleurs la plupart des activités rituelles, là aussi que les hommes se retrouvent chaque soir peu après le coucher du soleil pour discuter de choses et d'autres.

10. Pirarara en portugais. NdT.

11. Artíndia est un programme officiel de vente d' « artisanat indigène brésilien » de la Fondation Nationale de l'Indien (FUNAI), organisme du ministère de la Justice dont le siège se trouve à Brasília. Ce programme, qui compte des boutiques dans plusieurs capitales d'État au Brésil, est le principal revendeur d'« artisanat indigène » du pays. 
12. Le terme " authentique » (original en portugais, $\mathrm{NdT}$ ), catégorie sémantique « nativisée » par les Wauja, qualifie les objets et les performances produits dans des contextes rituels ou solennels dont la rigueur esthétique est directement liée à la honte et au respect manifestés envers les personnes qui recevront ces objets comme paiement rituel ou envers ceux qui assisteront aux performances. La catégorie « authentique » s'oppose à celle de paraguai, péjorative, qui s'applique aux faux, aux objets de mauvaise qualité et à l'artisanat destiné aux touristes. (Au Brésil, les objets estampillés made in Paraguai sont réputés de mauvaise qualité. NdT.)

13. Un bref rappel sur les tractations autour de l'acquisition des grands masques Atujuwá. En 1887, Karl von den Steinen voulut en rapporter un exemplaire pour le musée d'ethnologie de Berlin, mais des conditions logistiques précaires l'en empêchèrent. Finalement, en 1898, Herrmann Meyer y parvint à grand-peine, pour le même musée, mais cet exemplaire unique disparut en 1943 pendant le bombardement de la ville. Il fallut attendre presque un siècle pour qu'un masque Atujuwá soit acquis par un musée. En 1983, Emilienne Ireland ne parvint pas à en acheter une paire pour le National Museum of Natural History de la Smithsonian Institution de Washington. En 1994, Michael Heckenberger réussit à acheter une paire d'Atujuwá faits par les Kuikuru pour le Carnegie Museum of Natural History de Pittsburgh. La paire d'Atujuwá yanumaka fabriquée rituellement par les Wauja pour guérir Atamai en août 1997 a été vendue par la boutique Artíndia de Brasília à un antiquaire (Baú-Baú) de Salvador. Ces deux masques se trouvent certainement aujourd'hui dans une collection particulière dont l'identification ne devrait pas poser de problème. En juillet-août 2000, j'ai acquis pour le compte du Museu Nacional de Etnologia de Portugal un ensemble de trente-deux masques rituels en plus de deux paires de masques Atujuwá faits par les Wauja. Enfin, en mars 2005, les Wauja ont fabriqué trois paires d' Atujuwá pour le musée du quai Branly. À ce jour, aucun musée brésilien ne possède donc encore de grand masque Atujuwá.

14. Comparée au reste de l'Amazonie indigène, la culture matérielle wauja est assez sophistiquée et diversifiée. Il existe vingt-deux types de récipients en céramique (d'après la classification indigène), plusieurs types de vannerie pour la pêche, le transport et le stockage, des dizaines de sortes de masques, des clarinettes, des trompettes, des rhombes, des flûtes de bois et des troncs évidés (ces deux derniers objets sont considérés comme rares en Amazonie). Dans le système des objets xinguaniens, les Wauja sont les seuls à fabriquer les grandes marmites de céramique et les plaques à cuire les galettes de manioc qui sont utilisées comme paiements lors des rituels intra et inter-ethniques (Barcelos Neto 2000, 2004a).

15. La dernière ne fut utilisée qu'en septembre 2002.

\section{AUTEURS}

\section{ARISTÓTELES BARCELOS NETO}

Chercheur post-doctorant au département d'anthropologie de l'université de São Paulo, apapatai@usp.br 